Cahiers de recherches médiévales

Journal of medieval studies

$18 \mid 2009$

Le système d'enseignement occidental $\left(\mathrm{XI}^{\mathrm{e}}-\left.\mathrm{XV}\right|^{\mathrm{e}}\right.$ siècle)

\title{
Les maîtres parisiens et la genèse de l'Université (1200-1231)
}

Nathalie Gorochov

\section{CpenEdition}

Journals

Édition électronique

URL : https://journals.openedition.org/crm/11684

DOI : $10.4000 / \mathrm{crm} .17684$

ISSN : 1955-2424

Éditeur

Honoré Champion

Édition imprimée

Date de publication : 20 novembre 2009

Pagination : 53-73

ISSN : $1272-9752$

Référence électronique

Nathalie Gorochov, «Les maîtres parisiens et la genèse de l'Université (1200-1231) », Cahiers de recherches médiévales [En ligne], 18 | 2009, mis en ligne le 15 décembre 2012, consulté le 15 décembre 2022. URL : http://journals.openedition.org/crm/11684; DOI : https://doi.org/10.4000/crm.11684 


\section{㮣M}

\section{Les maîtres parisiens et la genèse de l’Université (1200-1231)}

Abstract: It was in the first quarter of the 13th century, particularly between 1208 and 1215 , that the Parisian schools developed into a full-fledged university complete with the requisite institutions and privileges. Interpretations of this relatively rapid phenomenon have so far relied on mainly general factors such as the discovery of a new Aristotle in the West (H. Grundmann), the opening of new careers to magistri (P. Classen), the will of the crown or the papacy (J. Verger) or the desire for reform of the schools (S. C. Ferruolo). This study, which is based on a prosopographic file of some 150 masters known to have taught in Paris between 1200 and 1231, develops the hypothesis that the birth of the university is closely linked to human factors peculiar to the Parisian schools. In effect we are also likely to find among the determining factors the social profile of the masters in the Capetian capital.

Résumé : C'est dans le premier quart du XIII siècle, en particulier entre 1208 et 1215, que les écoles parisiennes se sont muées en une université dotée de l'essentiel de ses institutions et privilèges. Pour interpréter ce phénomène relativement rapide, les spécialistes ont jusqu'à présent invoqué des facteurs assez généraux, tels que la découverte du nouvel Aristote en Occident (H. Grundmann), les nouvelles carrières ouvertes aux magistri (P. Classen), la volonté des pouvoirs royal et pontifical (J. Verger) ou une volonté de réforme des écoles (S. C. Ferruolo). S'appuyant sur un fichier prosopographique de quelque cent cinquante maîtres connus pour avoir enseigné à Paris entre 1200 et 1231, cette étude développe l'hypothèse selon laquelle la naissance de l'université de Paris est étroitement liée à des facteurs humains propres aux écoles parisiennes. En effet, le profil social des maîtres dans la capitale capétienne a sans doute été également déterminant.

L'université de Paris existait assurément, lorsque le pape Innocent III lui a adressé sa lettre Ex litteris vestris ${ }^{1}$, entre le début de l'année 1208 et la fin de l'année $1209^{2}$. Elle était déjà dotée de règlements, qui furent remplacés en 1215 par de

\footnotetext{
${ }^{1}$ Pour tous les documents cités, du privilège royal de 1200 à la bulle Ex litteris vestris, pour les statuts de 1215 et la bulle Parens Scientiarum de 1231, on se reportera à l'édition de H. Denifle et E. Châtelain, Chartularium Universitatis Parisiensis, t. 1 (1200-1289), Paris, 1889.

${ }^{2}$ L'historiographie depuis H. Denifle, Die Entstehung der Universitäten des Mittelalters bis 1400, I, Berlin, 1885 admet que cette lettre est le plus ancien document attestant l'existence de l'Université. Voir également S. C. Ferruolo, The origins of the University: the Schools of Paris and their Critics 1100-1215, Stanford, 1985; G. Post, «Parisian Masters as a Corporation 1200-1246», Speculum, 9, 1934, p. 423-450 ; les ouvrages de synthèse de J. Verger, Les universités au Moyen Age, Paris, 1973, rééd. 2000 et L'essor des universités au XIII ${ }^{e}$ siècle, Paris, 1997, ainsi que ses deux articles portant sur la naissance de l'université de Paris : «Des écoles à l'Université : la mutation institutionnelle» dans La France de Philippe Auguste. Le temps des mutations, éd. R.-H. Bautier, Paris, 1982, p. 817-846 et « À propos de la naissance de l'université de Paris : contexte social, enjeu politique, portée intellectuelle»,
}

Cahiers de Recherches Médiévales, 18, 2009 
nouveaux statuts rédigés par Robert de Courson. Dans les années suivantes, ses institutions se sont peu à peu mises en place jusqu'au règlement issu de la grande grève, confirmé par la bulle pontificale Parens Scientiarum en 1231. Ainsi, par une «mutation institutionnelle» assez rapide, somme toute, les écoles de Paris étaient devenues une université. Comme Jacques Verger l'a souligné, l'interprétation historique de ce phénomène soulève des difficultés. Doit-on le rattacher à la découverte du nouvel Aristote, comme le suggérait l'historien allemand Herbert Grundmann ${ }^{3}$, à une volonté de réforme des écoles ${ }^{4}$, à un éventail nouveau de carrières ouvertes aux magistri, autrement dit une sorte de demande sociale ${ }^{5}$, au rôle essentiel des pouvoirs, royal et surtout pontifical, selon Jacques Verger, qui insistait sur la conjonction probable de tous ces facteurs culturels, politiques et aussi de facteurs « humains $»^{6}$ ?

C'est ce dossier déjà épais sur la genèse de l'université de Paris que je me propose de rouvrir ici, à la lumière, cette fois, de données prosopographiques ${ }^{7}$ pour tenter de répondre aux questions suivantes : quels sont les maitres parisiens qui ont vécu la naissance de l'Université, qui ont accompagné sa formation ou ont pu au contraire la ralentir? Que sait-on de leurs origines géographiques, sociales, de leurs région et milieu d'origine, de leur lieu d'études, de leur carrière et de leur œuvre ? Ces facteurs et la personnalité des maîtres actifs à Paris entre 1200 et 1231 ont-ils pu être déterminants dans la genèse d'une université précisément à Paris, dans ces années-là ? Près de cent vingt clercs sont à ce jour connus pour avoir tenu école à Paris entre 1200 et 1231 : leurs profils, partiellement dévoilés, nous livrent certains éléments de réponses aux questions posées ci-dessus. Si la diversité du recrutement géographique était déjà perçue par les contemporains - Jacques de Vitry par exemple l'évoque dans un fameux et pittoresque passage de l'Historia Occidentalis $^{8}-$, l'étude prosopographique fait plus précisément ressortir des nations

initialement paru dans Schulen und Studium im sozialen Wandel des hohen und späten Mittelalters, éd. J. Fried, Sigmaringen, 1986, p. 69-96, réédité dans Les Universités françaises au Moyen Âge, Leyde, 1995, p. 1-35. Pour tous les travaux relatifs aux débuts de l'université de Paris antérieurs à 1980, il faut se reporter à S. Guenée, Bibliographie de l'histoire des universités françaises des origines à la Révolution, t. 1, Paris, 1981.

${ }^{3}$ H. Grundmann, Vom Ursprung der Universität im Mittelalter, 2e éd., Darmstadt, 1964.

${ }^{4}$ C'est l'hypothèse développée par S. Ferruolo, op. cit., p. 265 sq.

${ }^{5}$ P. Classen, «Die Hohen Schulen und die Gesellschaft im 12. Jahrhundert», Archiv für Kulturgeschichte, 48, 1966, p. 155-180.

${ }^{6}$ Dans les articles cités, note 2.

${ }^{7}$ Des données prosopographiques que j’ai pu rassembler depuis plusieurs années sur le milieu scolaire parisien du XIII ${ }^{\mathrm{e}}$ siècle et que je livre et commente plus amplement dans un mémoire $\mathrm{d}$ 'habilitation en cours de rédaction sur l'université de Paris et ses collèges au XIII ${ }^{\mathrm{e}}$ siècle.

${ }^{8}$ Jacques de Vitry, Histoire occidentale, traduction française par G. Duchet-Suchaux, introduction de J. Longère, Paris, 1997, p. 85-86 : «Non contents de s'affronter oralement en raison de positions divergentes, ou lors de Disputationes, ils [les étudiants] se querellaient, se jalousaient, se dénigraient entre eux en raison de la diversité de leurs nations, se lançant à la tête sans retenue un grand nombre d'injures et de propos outrageants, dénonçant les Anglais comme des ivrognes, des «coués », affirmant que des Français étaient des orgueilleux, des chiffes molles qui se paraient comme des femmes. Quant aux Teutoniques, ils disaient d'eux 
de poids, notamment les Anglais ou les Italiens, qui ont pu avoir une influence dans la corporation parisienne naissante, ce qui fera l'objet de la première partie de ce travail. Un autre apport de la prosopographie met en évidence, parmi les maîtres d'avant 1231, un assez fort pourcentage de nobles, examiné dans une deuxième partie. Leur présence a peut-être engendré des choix institutionnels particuliers, telle est l'hypothèse développée dans la troisième partie.

\section{Des maîtres venus de toute l'Europe: l'éventail des influences}

C'est un lieu commun dans l'historiographie consacrée aux universités médiévales : aux $\mathrm{XII}^{\mathrm{e}}$ et $\mathrm{XIII}^{\mathrm{e}}$ siècles, un peu moins par la suite, les scolares quittent leur région natale pour aller étudier puis voyager d'un centre d'enseignement à l'autre'. Au cours de cette peregrinatio academica encore fréquente et lointaine au $\mathrm{XIII}^{\mathrm{e}}$ siècle, beaucoup d'entre eux ont rencontré des modèles politiques, sociaux, académiques, culturels et ils ont pu tenter d'appliquer au moins partiellement ces modèles à l'institution universitaire naissante.

Ainsi les Anglais représentent la «nation $»^{10}$ la plus nombreuse parmi les maîtres parisiens de 1200 à 1231 et la qualité du travail d'A. B. Emden ${ }^{11}$, qui livre quantité de noms de maîtres parisiens, ne suffit pas à justifier ce poids. Un tiers des maîtres ayant enseigné à Paris entre 1200 et 1231 sont des Anglais. Pour John W. Baldwin, cet afflux d'Anglais dans les écoles de Paris tient à la valeur accordée aux études par le pouvoir royal et l'Église d'Angleterre qui emploient volontiers les magistri depuis le milieu du XII ${ }^{\mathrm{e}}$ siècle $^{12}$. Pourtant, les écoles d'Oxford se développent à partir de 1170-1180 en relation avec les tribunaux. Oxford, devenue résidence royale, accueille un important centre judiciaire dont les juristes savants commencent à partager leur temps entre la pratique dans les tribunaux et

qu'ils étaient des furieux, obscènes de surcroît dans leurs banquets. Les Normands pour leur part étaient des gens vaniteux et vantards, les Poitevins des traitres, amis des richesses. Quant à ceux qui étaient originaires de Bourgogne, ils leur faisaient la réputation de lourdauds et de sots. Jugeant de même les Bretons inconsistants et instables, ils leur reprochaient souvent la mort d'Arthur. Les Lombards, ils les disaient cupides, pleins de malignité et sans énergie, les Romains séditieux, violents et médisants, les Siciliens tyranniques et cruels, les Brabançons sanguinaires, incendiaires, brigands et voleurs, les Flamands prodigues, trop épris de beuveries, mous comme du beurre et apathiques. En raison même de ces invectives, ils abandonnaient souvent l'usage de la parole pour en venir aux mains ".

${ }^{9}$ J. Verger, «La mobilité étudiante au Moyen Âge », Histoire de l'éducation, 50, 1991, p. 65 90.

${ }^{10}$ Les nations n'ont pas encore d'existence institutionnelle à Paris à cette date, voir P. Kibre, The Nations in the Mediaeval Universities, Cambridge (Massachussetts), 1948, p. 65 sq.

${ }^{11}$ A. B. Emden, A Biographical Register of the University of Oxford to A.D. 1500, 3 vol., Oxford, 1957.

12 J. W. Baldwin, «Masters at Paris from 1179 to 1215. A social Perspective », dans Renaissance and Renewal in the Twelfth Century, éd. R. L. Benson et G. Constable, Cambridge (Massachussetts), 1982, p. 138-172, et du même auteur, "Studium et Regnum. The penetration of university personnel into French and English Administration at the turn of the twelfth and thirteenth Centuries », Revue des Etudes Islamiques, 44, 1976, p. 199-215. 
l'enseignement dans les écoles. À Oxford, on peut étudier le droit romain aussi bien que les arts et la théologie ${ }^{13}$. Il n'empêche que l'attrait pour Paris ne faiblit pas au tournant des $\mathrm{XII}^{\mathrm{e}}$ et $\mathrm{XIII}^{\mathrm{e}}$ siècles, les Anglais y étudient puis y enseignent parfois plusieurs années avant de repartir outre-Manche. Ajoutons que la proportion d'Anglais parmi les maîtres parisiens est plus forte pour les juristes et pour les artiens. Des seize maîtres connus pour avoir enseigné le droit canon et le droit civil à Paris entre 1200 et $1231^{14}$, quatre sont anglais, six sont originaires du diocèse de Chartres, alors que l'on ne compte à cette époque aucun maître en droit originaire de France du Nord ou de l'Est. Les Anglais se distinguent aussi dans les arts libéraux, représentant vingt-quatre maîtres ès arts sur une soixantaine recensés ${ }^{15}$, soit plus du tiers des artiens, parmi lesquels les premiers commentateurs de l'œuvre d'Aristote, John Blund par exemple. Une vingtaine de maîtres picards, champenois, flamands et tournaisiens - venus d'une zone qui préfigure la future nation picarde - enseignent la théologie entre 1200 et 1231 sur soixante-deux maîtres en théologie connus ${ }^{16}$, mais on ne trouve que douze théologiens anglais. Ainsi, la France du Nord et de l'Est fournit les plus gros effectifs de théologiens de même que les confins orientaux du royaume de France avec des maîtres venus de Basse Bourgogne, des Alpes, quelques Italiens, tandis que le sud-ouest du royaume demeure et pour longtemps encore largement sous-représenté.

Selon leur origine géographique, les maîtres ont reçu une première formation qui a pu orienter le choix de leur discipline. Par exemple dans les milieux de clercs chartrains, depuis Yves de Chartres $(\dagger 1116)$ semble subsister un goût persistant pour l'étude du droit ${ }^{17}$. Les maîtres passés à Oxford avant de venir étudier et enseigner à Paris ont, eux aussi, baigné dans un milieu favorable aux études juridiques. À l'inverse, beaucoup de maîtres formés dans des écoles cathédrales du nord et de l'est du royaume, ont été préparés à l'étude des arts libéraux et de la théologie, dans des milieux cléricaux plus étrangers voire hostile au droit. À ce titre, Étienne de Tournai ( $† 1203$ ), qui est l'un des premiers commentateurs français du Décret de Gratien, serait-il l'exception qui confirme la règle ? Avec les Anglais et les maîtres venus d'une large moitié nord et est du royaume de France, les arts libéraux et la théologie sont les disciplines dominantes au début du XIII ${ }^{\mathrm{e}}$ siècle, tandis que le droit représente, malgré les Anglais, un petit nombre d'écoles, encore réduit après la bulle Super Speculam de 1219 qui interdit l'enseignement du droit romain à Paris. Quant à la médecine, représentée par Gilles de Corbeil et Laurent de Fougères $^{18}$, son enseignement reste alors très marginal, semble-t-il.

\footnotetext{
${ }^{13}$ Sur les écoles d'Oxford au XII ${ }^{\mathrm{e}}$ siècle, la meilleure synthèse est celle de R. W. Southern, «From Schools to University», dans The History of the University of Oxford. Vol. 1: The Early Oxford Schools, éd. T. H. Aston, Oxford, 1984, p. 1-36.

${ }^{14}$ On voudra bien se reporter à la liste de brèves notices prosopographiques située en annexe.

${ }^{15} \mathrm{Cf}$. annexe.

${ }^{16} \mathrm{Cf}$. annexe.

${ }^{17}$ C'est la thèse présentée par E. Clerval, dans Les écoles de Chartres au Moyen Age, du Ve au $X V I^{e}$ siècle, Chartres, 1895, lorsqu'il évoque, p. 319, l'étude du droit à Chartres dans la seconde moitié du XII ${ }^{\mathrm{e}}$ siècle.

${ }^{18} \mathrm{Cf}$. annexe. Sur les origines de l'enseignement de la médecine à Paris, voir les premières pages du livre de D. Jacquart, La médecine médiévale dans le cadre parisien, Paris, 1998.
} 
Les groupes nationaux les plus nombreux peuvent donc avoir influé sur les disciplines enseignées mais également sur des choix institutionnels de la nouvelle université. Ainsi, selon les auteurs de l'irremplaçable History of the University of Oxford, en particulier R. W. Southern et M. B. Hackett ${ }^{19}$, le contrôle du prix des logements occupés par les scolares existait bel et bien dans les écoles oxfordiennes dès le début du XIII ${ }^{\mathrm{e}}$ siècle, avant la grève de 1209 et bien avant que ce système ne soit mentionné dans des documents parisiens. N'est-il pas légitime de supposer que les maîtres anglais ont importé à Paris ce système qui empêchait les propriétaires de louer à un prix exorbitant leurs logements aux gens des écoles ? De même, la présence d'Italiens - une dizaine de maîtres attestés - a dû jouer dans la procédure élective mise en place dès le début de l'université de Paris dans les assemblées de maîtres. Rappelons que le droit romain, amplement étudié et connu dans les milieux scolaires depuis le $\mathrm{XII}^{\mathrm{e}}$ siècle, avait posé plusieurs principes régissant la vie des communes ou les métiers ${ }^{20}$. Ainsi avait-il établi la nécessité pour un groupe d'agir par l'intermédiaire d'un représentant ou délégué élu, mais aussi de procéder à des élections qui expriment la volonté de tous. Les civilistes avaient au XII ${ }^{\mathrm{e}}$ siècle produit des textes concernant les modalités des élections, de même que les canonistes pour réglementer les élections dans l'Église ${ }^{21}$. Dès les premières années du XIII ${ }^{\mathrm{e}}$ siècle, ces modèles élaborés par les juristes ne sont pas ignorés des maîtres parisiens, tous membres de l'Église, parmi lesquels se trouvent : des Italiens issus du monde communal, comme le célèbre chancelier Prévotin de Crémone ou maître Pierre de Capoue ${ }^{22}$; des canonistes bons connaisseurs du droit romain comme Petrus Brito, un éminent décrétaliste parisien entre 1205 et $1218^{23}$; des civilistes dont le membre le plus éminent, l'Anglais Pierre Peverel, promu évêque d'Agde en 1214,

\footnotetext{
${ }^{19}$ The History of the University of Oxford. Vol. 1 : The Early Oxford Schools, éd. T. H. Aston, Oxford, 1984, cf. en particulier les deux premiers chapitres par R. W. Southern, cité plus haut, et par M. B. Hackett, «The University as a Corporate Body», p. 37-95.

${ }^{20}$ Comme le montre P. Michaud-Quantin dans Universitas. Expressions du mouvement communautaire dans le Moyen Âge latin, Paris, 1970.

${ }^{21}$ "Ce qui intéresse tout le monde doit recevoir l'approbation de tous », Code 5.59.5, une formule utilisée depuis le deuxième quart du $\mathrm{XIII}^{\mathrm{e}}$ siècle pour mettre en œuvre des organismes représentatifs, selon P. Michaud-Quantin, op. cit., p. 325, qui reprend G. Post, « A Romano-Canonical Maxim : quod omnes tangit ", Traditio, 4, 1946, p. 197-252 ainsi que Y.M. Congar, "Quod omnes tangit tractari et approbari debet", Revue historique du droit français et étranger, 1958, p. 210-249. Sur les modalités juridiques des élections, voir P. Michaud-Quantin, op. cit., chapitre V : Expressions de la volonté collective, p. 271-284, ainsi que les publications antérieures à sa synthèse : A. Esmein, «L'unanimité et la majorité dans les élections canoniques », dans Mélanges H. Fitting, Montpellier, 1907, p. 357-382; E. Ruffini-Avondo, I sistemi di deliberazione collettiva nel medioevo italiano, Turin, 1927 ; L. Moulin, "Sanior et major pars. Note sur l'évolution des techniques électorales dans les ordres religieux du VI ${ }^{\mathrm{e}}$ au XIII' siècle », Revue historique du droit français et étranger, 1958, p. 368-397 ; J. Gaudemet, «Unanimité et majorité », dans Mélanges N. Didier, p. 149-162, Paris, 1960.

${ }^{22} \mathrm{Cf}$. annexe.

${ }^{23} \mathrm{Cf}$. annexe.
} 
est de fait éloigné de Paris, cinq ans avant l'interdiction de Super Speculam ${ }^{24}$; et bien sûr des maîtres qui ont étudié voire enseigné quelques années à Bologne ${ }^{25}$ et qui ont pu assister, là-bas, aux élections de recteur attestées vers $1200^{26}$.

Qu'ils soient italiens, juristes, anciens étudiants de l'université de Bologne, tous ceux que l'on vient de citer ont sans aucun doute joué un rôle éminent dans la mise en place de procédures électives afin de désigner des procureurs de l'université de Paris, dès les années 1208-1209². Mais il faut attendre 1230 environ pour que le rectorat bolonais soit imité à Paris, et que les universitaires parisiens prennent l'habitude de désigner un représentant plus régulier, le recteur ${ }^{28}$. Ils adaptent leur modèle à la situation parisienne, réservant l'élection du recteur aux seuls docteurs. Au même moment à Bologne, si l'institution rectorale est bien établie, les docteurs acceptent mal d'être exclus du processus électif. Ainsi, Odofredo, célèbre docteur en droit bolonais, comparant le cas bolonais au cas parisien, remarque dans l'un de ses commentaires qu'à Bologne, seuls les docteurs devraient élire le recteur, à l'instar de ce qui se passe à Paris ${ }^{29}$. Ainsi l'on tente de s'inspirer du modèle parisien à Bologne si bien que l'on peut parler d'influences réciproques. La brièveté du mandat du recteur, d'un mois seulement, ne laisse pas le temps à cet élu d'exercer une autorité sur ses confrères, attentifs à préserver leur autonomie, d'autant plus attentifs que l'aristocratie, qui a vocation naturelle à commander, fournit dans le premier tiers du $\mathrm{XIII}^{\mathrm{e}}$ siècle un assez fort pourcentage de maîtres susceptibles d'être élus, un jour ou l'autre, en tant que chefs de la corporation, et de nuire au principe d'égalité entre les membres jurés.

\section{Le poids non négligeable des maîtres nobles}

Il y a une trentaine d'années, l'historien américain John W. Baldwin a esquissé le portrait social de quarante-sept maîtres parisiens du règne de Philippe Auguste (1180-1223) qu'il avait pu repérer lors de ses recherches sur le cercle de Pierre le Chantre: il les présentait comme des hommes plutôt issus de la petite noblesse rurale que de la bourgeoisie urbaine ou de la haute aristocratie ${ }^{30}$. Dans l'échantillon un peu plus large dont on dispose aujourd'hui, près de $25 \%$ des maîtres

\footnotetext{
${ }^{24} \mathrm{Cf}$. annexe.

${ }^{25}$ Par ex., Nicolas de Fernham, Nicolas Haudry de Chartres, Richard de Mores, cf. annexe.

${ }^{26}$ O. Weijers, Terminologie des universités au XIII siècle, Rome, 1987, p. 187.

${ }^{27}$ Sur les procédures électives dans l'Université, je me permets de renvoyer à l'article suivant: N. Gorochov, J. Verger, «Les élections dans le monde universitaire au Moyen Âge », dans Elections et pouvoirs politiques du VIIe au XVIIe siècle, C. Peneau dir., Bordeaux, 2008, p. 121-142.

${ }^{28}$ Selon O. Weijers (op. cit., p. 188), la première attestation du mot se trouve dans la bulle Parens Scientiarum (1231).

${ }^{29}$ Ipsi doctores qui exercent professionem, debent eligere rectores. Et ita dicitur quod est Parisius, quod doctores eligunt rectores et non scolares. Cité par O. Weijers, op. cit., p. 188.

${ }^{30} \mathrm{~J}$. W. Baldwin, «Masters at Paris... », note 12. En réalité, J. Baldwin y mène précisément l'enquête sur les années 1180-1215. Sur le cercle de Pierre le Chantre, il faut naturellement voir J. W. Baldwin, Masters, Princes and Merchants. The social Views of Peter the Chanter and his Circle, Princeton, 1970.
} 
sont issus de la noblesse, ce qui corrobore les résultats de John Baldwin ${ }^{31}$, et l'aristocratie est incontestablement sur-représentée. Mais quelle aristocratie? Pour mieux la cerner, examinons par exemple les maîtres nobles qui enseignent à Paris en 1206. Parmi les théologiens, on rencontre Étienne Langton, qui enseigne jusqu'en 1206, fils d'Henri Langton, un membre de la gentry habitant un manoir près de Wragby en Lincolnshire ${ }^{32}$; Guillaume de Joinville, fils du sénéchal Geoffroy IV de Joinville, qui enseigne la théologie jusqu'à son élection au siège épiscopal de Châlons en 1208; Humbert de Pirovano, membre d'une éminente famille de l'aristocratie milanaise, qui en 1206 quitte Paris pour Milan dont il vient d'être élu archevêque; Robert de Courson, dont la famille implantée dans le Derbyshire est proche de la famille royale anglaise et qui enseigne la théologie à Paris jusqu'à son accession au cardinalat en 1212; Pierre de Capoue, qui appartient à une famille romaine en pleine ascension et quitte d'ailleurs les écoles parisiennes lorsqu'il est promu cardinal par Honorius III en 1219; Adam de Courlandon, frère de Renaud, seigneur de Courlandon, vassal du seigneur de Nesle, en Picardie, qui est né dans une famille châtelaine. Parmi la probable centaine de maîtres ès arts, notons la présence de Grégoire de Crescenzi, issu de l'aristocratie romaine, qui enseigne à Paris entre 1200 et 1216 , neveu d'un cardinal d'Innocent III, lui-même promu cardinal en 1216, d'Eudes de Cheriton, un noble anglais qui porte le nom du manoir familial situé dans le Kent, des frères Aubry et Gautier Cornut, seigneurs du Chartrain, tous deux étudiants puis maitres ès arts (et sans doute en droit, pour Aubry) dans le premier quart du XIII ${ }^{\mathrm{e}}$ siècle. Ainsi se côtoient des maîtres issus de l'aristocratie romaine, de la gentry anglaise et de la petite et moyenne noblesse française.

Est-ce l'amour désintéressé du savoir et /ou l'ambition sociale qui conduisent ces cadets de familles nobles aux écoles de Paris? Bien qu'ils n'eussent jamais confessé de tels sentiments, certains de ces maîtres ont sans conteste été animés par la curiosité, l'intérêt, voire la passion pour la discipline qu'ils ont parfois enseignée pendant de longues années. Ainsi, le noble romain Grégoire de Crescenzi, dont l'entrée au Sacré Collège était assurée, quel que fût son cursus antérieur, par l'entremise d'un oncle cardinal, s'est manifestement plu à enseigner une dizaine d'années à Paris. Parmi les maîtres de 1206 que l'on vient de citer, quelques-uns «à qui il suffit de naître» et qui ont «la prélature dans leur berceau $»^{33} \mathrm{~s}$ 'appliquent à

\footnotetext{
${ }^{31} 27$ sur presque 120 , pour être exact, 27 sur 50 dont les origines sociales sont connues. Les nobles représentent donc au moins $23 \%$ de tous les maitres. Naturellement, on peut conjecturer que les origines nobles sont plus systématiquement signalées dans la documentation et s'en tenir à une trentaine de nobles sur 120 maîtres. A contrario, la noblesse ou du moins la richesse peuvent en certains cas être dissimulées, ainsi l'hagiographe de saint Edmond d'Abingdon, archevêque de Canterbury et ancien maître parisien, insiste sur la modestie de ses origines, alors que ses parents, les Le Rich d'Abingdon, possédaient d'importants biens fonciers, cf. annexe.

${ }^{32}$ Pour Étienne Langton et tous les maîtres cités à la suite dans ce paragraphe, cf. annexe.

${ }^{33}$ B. Guenée, (Entre l'Église et l'Etat. Quatre vies de prélats français à la fin du Moyen Âge, Paris, 1987, p. 21), distingue deux types de carrières, deux types de prélats, « ceux auxquels il suffisait de naître » et « ceux qui n'avaient pas la prélature dans leur berceau », « les premiers
} 
étudier jusqu'à tenir école alors qu'ils ont à portée de main les bénéfices ecclésiastiques les plus convoités - s'ils ne les détiennent déjà pendant leurs études. Est-ce donc qu'au tournant des $\mathrm{XII}^{\mathrm{e}}$ et XIII ${ }^{\mathrm{e}}$ siècles, les élites - le haut clergé en particulier-, reconnaissent la valeur du savoir scolaire et que les clercs ambitieux ont conscience que les études offrent une large voie d'accès aux dignités ?

Pour l'historien allemand Peter Classen, c'est incontestablement le pouvoir pontifical qui fut le premier, dès le XII ${ }^{\mathrm{e}}$ siècle, à saisir l'intérêt que la Curie et, de manière générale, l'Église, avaient à recruter des clercs passés par les écoles de théologie ou de droit canon ${ }^{34}$. À partir des années 1140, quelques clercs italiens, formés dans les écoles de Paris, Laon, Chartres ou Bologne, entrés au Sacré Collège ou montés sur le trône de Saint-Pierre, tels l'ami et peut-être disciple d'Abélard, Guido da Città di Castello, «l'homme le plus savant de Rome $»^{35}$, promu cardinal en 1128 puis pape sous le nom de Célestin II (1143-1144), Rolando de Sienne, théologien et canoniste de Bologne, chancelier de l'Église romaine puis pape sous le nom d'Alexandre III (1159-1181), ou bien encore le cardinal Hyacinthe, un fervent partisan d'Abélard, membre du Sacré Collège de 1144 à 1191 et finalement élu sous le nom de Célestin III (1191-1198), ont, au moins par leur parcours ${ }^{36}$, diffusé dans les milieux curiaux l'idée nouvelle que l'Église devait prioritairement promouvoir les théologiens et les canonistes, anciens étudiants ou maîtres, dont le savoir serait ainsi efficacement mis au service de la doctrine, de l'administration ecclésiastique ou encore de la pastorale. L'Église romaine devait tirer parti de l'extraordinaire essor des écoles du XII ${ }^{\mathrm{e}}$ siècle.

D'où l'afflux précoce des nobles romains et italiens aux écoles de Paris, un afflux assez visible dès le milieu du XII ${ }^{\mathrm{e}}$ siècle, comme en témoigne un recueil épistolaire constitué à Saint-Victor de Paris, rempli de lettres des pères, oncles, frères des étudiants italiens adressées à des prélats français chargés de protéger et d'aider lesdits écoliers ${ }^{37}$. Les familles de la noblesse romaine comme les Boboni, les Pierleoni et d'autres ${ }^{38}$, informées aussitôt de l'estime portée par la papauté et les

étaient de grands seigneurs ou personnages. Les seconds étaient issus de la petite noblesse ou de la bourgeoisie aisée [...] Leur carrière ne devait pas grand-chose à leur naissance ».

${ }^{34}$ P. Classen, «La curia romana e le scuole di Francia nel secolo XII », dans Le istituzioni della Societas Christiana dei secoli XI-XII. Papato, cardinalato e episcopato, Milan, 1974, p. $432-436$.

${ }^{35}$ Ibid..

${ }^{36}$ Peut-être aussi en favorisant, quand ils en avaient le pouvoir, l'entrée des maitres à la Curie et au Sacré collège mais, faute de sources, une telle hypothèse ne peut pas être vérifiée.

${ }^{37}$ P. Classen, op. cit. Voir aussi les lettres de recommandation en faveur d'étudiants italiens adressées par des consuls romains au roi Louis VII et publiées par Denifle et Châtelain dans le Chartularium, t. 1, $\mathrm{n}^{\mathrm{O}} 31,32,33,34$, p. 37-38.

${ }^{38} \mathrm{P}$. Classen a repéré parmi les étudiants parisiens Sasso, neveu du pape Anastase IV (11531154), un «Bobo», plus tard cardinal membre de la famille des Boboni, ou encore plusieurs fils Pierleoni, l'une des plus puissantes familles romaines de la seconde moitié du XII ${ }^{e}$ siècle. Vers 1170, Lothario dei Segni, futur Innocent III, fils de la petite noblesse du Latium, arrive à Paris pour étudier. Sur la jeunesse d'Innocent III, voir M. Maccarrone, «Innocent III prima del pontificato », Archivio della $r$. deputazione romana di storia patria, 1943, p. 59-134 ainsi 
cardinaux aux scolares, mènent dès le milieu du XII ${ }^{\mathrm{e}}$ siècle une «politique éducative cohérente visant à assurer à quelques-uns de leurs fils une carrière curiale $\aleph^{39}$ par une solide formation intellectuelle à Paris notamment. Et le flux des nobles italiens vers la capitale capétienne ne faiblit pas, au XIII ${ }^{\mathrm{e}}$ siècle, les familles de l'aristocratie romaine alors en pleine ascension, comme les Crescenzi ${ }^{40}$, les Conti ${ }^{41}$, les Colonna $^{42}$, - plus tard les Capocci, les Fieschi ou les Caetani et les nobles milanais de même tels les Pirovano - ayant bien conscience que la haute naissance, les relations, l'ambition ou la protection ne suffisent plus pour atteindre le sommet de l'Église désormais presque exclusivement réservé aux savants ${ }^{43}$. Ceci explique, dans le premier tiers du XIII ${ }^{\mathrm{e}}$ siècle, la présence à Paris, parmi les maîtres qui tiennent école, de nobles clercs du Latium ou de Lombardie cités plus haut, Humbert de Pirovano, Grégoire de Crescenzi etc. Selon Pascal Montaubin, ces aristocrates italiens ont pris «une certaine avance sur le reste du monde aristocratique européen $»^{44}$. Ainsi les pontifes et leur entourage ont-ils lentement instauré une sorte de méritocratie qui tenait compte de la valeur personnelle, culturelle des clercs, un mode de promotion immédiatement perçu par la noblesse romaine et celle plus largement italienne $\mathrm{e}^{45}$.

que K. Pennington, «The legal Education of Pope Innocent III », Bulletin of Medieval Canon Law, 1974, p. 70-77, rééd. dans Popes, Canonists and Texts, Londres, 1993, n 1.

${ }^{39} \mathrm{P}$. Montaubin, dans sa thèse inédite, Le gouvernement par la grâce. La politique bénéficiale des papes au XIII siècle dans la moitié nord de la France, Paris I, 1998, développe, dans la deuxième partie, les nouvelles stratégies familiales des familles italiennes pour faire entrer leur fils à la Curie dès le XII ${ }^{\mathrm{e}}$ siècle, en particulier leur politique éducative, p. 342 sq. P. Montaubin, examinant les principales familles qui émergent à Rome vers 1200, montre que certaines d'entre elles ne pratiquent pas systématiquement cette politique et ne réussissent pas l'ascension souhaitée, tandis que d'autres accèdent au rang de familles baronniales vers 1250 , comme les Conti ou les Colonna. Voir aussi sur ce point M. Venditelli, «Mercanti Romani del primo Ducento in Urbe Potentes ", dans Roma nei secoli XIII e XIV. Cinque Saggi, éd. E. Hubert, Rome, 1993, p. 87-135 ; S. Carocci, Baroni di Roma. Dominazioni signorili e lignaggi aristocratici nel Duecento e nel primo Trecento, Rome, 1993 ; M. Thumser, Rom und der römische Adel in den Späten Stauferzeit, Tübingen, 1995. Ces travaux montrent le profond renouvellement de la noblesse romaine vers 1200 : tandis que les Boboni et les Pierleoni déclinent, des familles de second rang connaissent une ascension spectaculaire entre 1200 et 1250, en prenant le contrôle du Sénat, en infiltrant la Curie : il s'agit des Orsini, des Conti, des Annibaldi, des Capocci, des Malabranca, des Colonna.

${ }^{40}$ Grégoire de Crescenzi, cf. annexe.

${ }^{41}$ Lothario dei Segni, de la famille des Conti, futur Innocent III, voir note 38.

${ }^{42}$ Giovanni di Giordano Colonna est étudiant à Paris vers 1226, selon P. Montaubin, op. cit., p. 408.

${ }^{43}$ Encore est-il trop souvent impossible d'affirmer, même au XIII ${ }^{\mathrm{e}}$ siècle, quel facteur l'emporte dans la collation pontificale des bénéfices ecclésiastiques : le népotisme, la volonté de financer les études, la rétribution de services rendus ou bien la valeur accordée aux grades universitaires, selon P. Montaubin, op. cit., p. 351.

${ }^{44}$ P. Montaubin, op. cit., p. 352 .

${ }^{45}$ Et plus tard explicitement invoqué dans les lettres de collations diffusées dans toute la Chrétienté au cours du XIII ${ }^{\mathrm{e}}$ siècle, P. Montaubin, op. cit., p.447 sq. 
Mais au-delà, en dehors de l'Italie, cette méritocratie trouve-t-elle une audience dès le XII ${ }^{\mathrm{e}}$ siècle, auprès des collateurs de bénéfices ecclésiastiques comme des clercs à la recherche de bénéfices? Les premiers sont-ils désormais prêts à fermer les yeux sur une origine sociale modeste pourvu que les seconds fussent des clercs savants? Faisant dans ses Digesta pauperis scolaris l'apologie des pauvres clercs formés aux écoles, le cardinal Albinus, un éminent membre de la Curie d'assez modeste extraction, offre vers 1180 des fondements théoriques à ce système méritocratique naissant ${ }^{46}$. Mais dans quelle mesure sa pensée a-t-elle été diffusée ? Du point de vue des clercs, la méritocratie n'a-t-elle pas pour effet d'encourager tous les ambitieux, quelle que soit leur extraction sociale et spécialement les plus modestes, ceux qui «n'avaient pas la prélature dans leur berceau », à venir se former dans les grands centres scolaires, les plus prestigieux étant Paris et Bologne après 1150, afin d'entrer plus facilement dans un chapitre cathédral, d'espérer en devenir un dignitaire et, pourquoi pas, d'accéder un jour à l'épiscopat voire au cardinalat ?

Dans le groupe des maîtres présents entre 1200 et 1231, les clercs nés dans des familles de la petite ou moyenne noblesse française et anglaise sont fort nombreux, on l'a vu plus haut; les plus âgés sont venus étudier à Paris vers 11801190. Il est donc probable que ces membres de la gentry et ces fils de petits seigneurs ruraux du nord de la France viennent aux écoles par espoir d'ascension sociale. C'est ce que confirment, pour les Anglais au moins, les résultats de Julia Barrow dans une étude comparative menée sur les chanoines anglais et allemands au $\mathrm{XII}^{\mathrm{e}}$ siècle et sur leur rapport aux études ${ }^{47}$. Selon cette historienne anglo-saxonne, si les clercs allemands voyageaient peu pour étudier au XII ${ }^{\mathrm{e}}$ siècle, sauf la très haute aristocratie, minoritaire ${ }^{48}$, c'est parce qu'ils étaient accueillis très jeunes dans des chapitres cathédraux près de chez eux où ils étaient formés à l'école cathédrale puis où ils restaient comme chanoines prébendés à l'âge adulte. Ce qui expliquerait le nombre relativement faible d'étudiants et de maîtres allemands à Paris à la fin du $\mathrm{XII}^{\mathrm{e}}$ comme dans le premier tiers du XIII ${ }^{\mathrm{e}}$ siècle $^{49}$. La faible monétarisation de la Germanie nuisait par ailleurs au financement de tels voyages d'études. Au contraire, en Angleterre comme en France, ce système de chanoines-écoliers n'existe nullement, les jeunes nobles voués à la cléricature partaient dès qu'ils le pouvaient vers Paris, ou dans une moindre mesure vers Bologne, bien conscients dès le XII

${ }^{46}$ U. R. Blumenthal, «Cardinal Albinus of Albano and the Digesta pauperis scolaris Albini, ms. Ottob. Lat. 3057 », Archivium Pontificae Historiae, 20, 1982, p. 7-50 et Th. MontecchiPalazzi, «Formation et carrière d'un grand personnage de la Curie au XII ${ }^{\mathrm{e}}$ siècle : le cardinal Albinus », Mélanges de l'École française de Rome, 98, 1986, p. 623-650.

${ }^{47} \mathrm{~J}$. Barrow, «Education and the Recruitment of Cathedral Canons in England and Germany (1100-1225)», Viator, 1989, p. 117-137.

${ }^{48}$ Par exemple les fils d'Henri le Lion. Cf. J. Elhers, «Deusche Scholaren in Frankreich während des 12. Jahrhunderts ", dans Schulen und Studium im sozialen Wandel des hohen und späten Mittelalters, éd. J. Fried, Vorträge und Forschungen 30, Sigmaringen, 1986, p. $97-$ 120. Quelques clercs Allemands, issus de la haute noblesse, viennent recevoir à Paris l'enseignement supérieur que les écoles cathédrales de Germanie ne leur offrent pas. De même, quelques clercs d'extraction plus modeste n'ont pas le choix : s'ils souhaitent devenir écolâtres, ils doivent se former à la théologie et au droit à Paris.

${ }^{49} \mathrm{C}$ 'est une des conclusions de Julia Barrow, op. cit. 
siècle que leur formation intellectuelle assortie d'un probable titre de magister les aiderait à recevoir une prébende canoniale ${ }^{50}$. La monétarisation plus élevée de ces deux royaumes facilite le paiement de tels voyages dans des milieux aisés. Et l'essor scolaire anglais plus tardif, après 1150 , n'infléchit pas la tradition de la peregrinatio academica en France des clercs anglais, plus forte que jamais vers 1200, comme l'atteste le fort pourcentage de maîtres anglais à Paris entre 1200 et 1231. L'espoir de mobilité sociale grâce aux études est sans doute d'autant plus grand chez les Anglais que le recrutement des chanoines, étroitement contrôlé par les évêques, reste plus ouvert que sur le continent donc plus favorable aux magistri, alors qu'en France, l'autorité des chapitres en matière de collation des prébendes a pour conséquence, au XII ${ }^{\mathrm{e}}$ siècle, un recrutement local privilégiant les relations plutôt que les études. Il n'empêche que, par leur renommée, par leur rayonnement exceptionnel, les écoles de Paris exercent déjà une attraction spectaculaire sur les nobles français qui constituent une bonne part des maîtres entre 1200 et 1231 .

Ainsi, depuis le milieu du $\mathrm{XII}^{\mathrm{e}}$ siècle, l'image du pasteur nécessairement lettré, doté d'une culture savante ou scolaire solide, cette image forgée au sein même de la curie romaine, s'est bien diffusée chez ces fils destinés à la cléricature nés dans l'aristocratie qu'elle soit romaine, milanaise, française ou anglaise. Cette image qui confère une grande valeur à l'activité intellectuelle au service de la pastorale semble avoir gagné des strates inférieures de la noblesse, jusqu'à la seigneurie châtelaine, au moins un petit nombre de familles de la noblesse, et en deçà de la noblesse, la bourgeoisie ainsi que des couches sociales plus modestes. Car il y a parmi les maittres entre 1200 et 1231 des fils de bourgeois, peu nombreux, deux fois moins nombreux que les nobles ${ }^{51}$. Enfin, ils ne sont que quatre sur cent vingt maitres d'origine plus modeste, nés dans des familles de la paysannerie ou de l'artisanat : le médecin de Philippe Auguste et maître en médecine Gilles de Corbeil ${ }^{52}$, Robert de Sorbon « fils de vilain et de vilaine $»^{53}$ et Guillaume de Saint-Amour, tous deux

\footnotetext{
${ }^{50}$ Voir aussi A. L. Gabriel, "English Masters and Students in Paris during the Twelfth Century », dans Garlandia. Studies in the history of the Mediaeval University, Notre Dame, Indiana, 1969, p. 1-37.

${ }^{51}$ Edmond et Robert d'Abingdon, Boniface de Bruxelles, Nicolas Haudri de Chartres, Étienne Bérout, Philippe de Grève, Richard de Fournival, Gautier de Marvis, Gautier de Wangiermès, Nicolas Buchiau de Tournai, cf. annexe.

${ }^{52} \mathrm{Cf}$. annexe.

${ }^{53}$ Selon le fameux passage de la Vie de saint Louis de Jean de Joinville, éd. J. Monfrin, Paris, 1995 , p. 14-19 où Joinville rapporte une discussion qu'il a eue avec Robert de Sorbon: «Maistre Robert, que me voulez-vous? Et me dist : Je vous veil demander se li roys se seoit en cest prael, et vous vous aliez seoir sur son banc plus haut que li, se on vous en devroit bien blasmer. Et je li diz que oil. Et il me dist : Donc faites vous à blasmer, quant vous estes plus noblement vestus que li roys ; car vous vous vestez de vair et de vert, ce que li roys ne fait pas. Et je li diz: Maistre Robert, sauve vostres grace, je ne faiz mie à blasmer se je me vest de vert et de vair ; car cest abit me lessa mes pères et ma mère. Mais vous faites à blasmer ; car vous estes fils de vilain et de vilainne, et avez lessié l'abit de vostre père et vostre mère, et estes vestus de plus riche camelin que li roys n'est. Et lors je pris le pan de son seurcot et dou seurcot le roy, et li diz : Or esgardez si je di voir. Et lors li roys emprist à deffendre maistre Robert de paroles, de tout son povoir. »
} 
maîtres ès arts dès $1220-1225$, puis maîtres en théologie ${ }^{54}$, enfin Jacques Pantaléon d'Ancher, fils d'un cordonnier de la ville de Troyes, le seul à s'être véritablement arraché à sa condition de non-noble en devenant pape sous le nom d'Urbain IV (1261-1264) $)^{55}$, aucun des trois précédents n'ayant en revanche accédé au rang d'évêque. Tous les quatre ont abondamment écrit, mais sans jamais évoquer, même par allusion, la curiosité intellectuelle ou l'espoir d'ascension sociale qui avaient pu les conduire aux écoles de Paris.

Ainsi, entre 1200 et 1231 , près d'un maître parisien sur quatre est noble ${ }^{56}$ et les données prosopographiques permettent d'affirmer qu'il existe d'assez forts contrastes socio-économiques entre les maîtres qui se côtoient au sein des écoles. Reste à mettre en évidence un hypothétique rapport entre cette configuration sociale et la mutation des écoles en université. Au sein de la corporation en voie d'organisation, le poids des nobles a-t-il pu représenter un frein ou bien ceux-ci ontils au contraire joué un rôle moteur? Les contrastes sociaux entre maîtres très riches et maîtres pauvres ont-ils engendré des tensions?

\section{Le profil social contrasté des maîtres et la nouvelle institution}

Lorsque Pierre le Chantre, puis ses disciples, discutent de la question des salaires ou dons versés par les étudiants aux maîtres, pour déterminer s'ils sont licites ou s'ils relèvent de la simonie, tous invoquent, pour justifier cette pratique, une réalité sociale qui leur semble familière, le cas des maîtres indigents57. Quand ils n'ont pas assez de revenus, les maîtres parisiens peuvent légitimement réclamer de l'argent à leurs étudiants, affirment ces théologiens. Faisant écho au Verbum Abbreviatum de Pierre le Chantre: Non nego tamen quin possint accipere modicum salarium si indigeant $^{58}$, Étienne Langton écrit dans ses Questiones: Si tamen indigeat licet ei accipere necessaria ab auditoribus suis sine expressa nominatione certe summe, et hoc propter scandalum ${ }^{59}$, tandis que Robert de Courson parvient à la même conclusion au terme d'un long passage de sa Summa ${ }^{60}$. Ces auteurs ont une conscience aiguë du fossé qui sépare les nobles maîtres riches et prébendés des maîtres sans bénéfice et sans revenus, parce que les situations matérielles contrastées des uns et des autres sont probablement bien visibles dans la ville dans les premières années du XIII ${ }^{\mathrm{e}}$ siècle.

Les sources de ces années-là ne contiennent guère de traces de tensions spécifiques entre les maîtres mais, à en croire Roger de Hoveden, il semble bien que la rixe quasi «fondatrice» de 1200 ait été déclenchée par une querelle entre un modeste tavernier parisien et le serviteur d'Henri de Jaceyo, un riche étudiant (ou

\footnotetext{
${ }^{54}$ M.-M. Dufeil, Guillaume de Saint-Amour et la polémique universitaire 1250-1259, Paris, 1972.

${ }^{55} \mathrm{Cf}$. annexe.

${ }^{56}$ Ce pourcentage est probablement supérieur à celui des étudiants nobles qui fréquentent les écoles parisiennes, mais il n'en est pas question ici.

${ }^{57}$ J. W. Baldwin, Masters, princes and merchants, vol. 1, p. 124 sq. et notes, vol. 2, p. 84 sq.

${ }^{58}$ Ibid., vol. 2 p. 85 note 63.

${ }^{59}$ Ibid., note 62 .

${ }^{60}$ Ibid., p. 85-87.
} 
maître) allemand, qui, accompagné d'autres nobles, a souhaité venger son domestique par une violente bagarre ${ }^{61}$. Analysant les conflits town and gown, Jacques Verger reconnaît que plusieurs d'entre eux ont été provoqués par des nobles qui ne perdaient ni leur arrogance, ni leur violence ou leur goût pour les armes lorsqu'ils venaient étudier ou enseigner dans une ville universitaire ${ }^{62}$. Par ailleurs, c'est peut-être le caractère très hétérogène du groupe des maîtres qui les contraint, dès qu'ils s'associent en université - et plus que toute autre organisation professionnelle urbaine-, à renforcer la cohésion de leur métier par des vêtements uniformes, à sauvegarder l'égalité entre les membres par une fréquente participation aux assemblées, à créer la solidarité entre les maîtres par l'assistance de tous aux funérailles de l'un d'eux ${ }^{63}$. S'inspirant probablement des autres métiers urbains qui s'organisent alors dans les villes d'Occident, les maîtres parisiens, cherchant à s'associer, à s'accorder, à se doter de statuts jurés, ont dû être confrontés aux forces à la fois centrifuges et hiérarchisantes qui traversaient nécessairement un groupe d'hommes aux origines géographiques et sociales si diverses.

Rien dans ce qui vient d'être dit ne permet de confirmer ou d'infirmer le rôle moteur des nobles dans l'institution naissante. Toutefois, lorsque à l'issue d'un long conflit avec le chancelier de l'Église de Paris, en 1212-1213, les maîtres obtiennent enfin du chancelier l'autonomie du recrutement, en lui imposant d'accorder à l'avenir la licencia docendi aux candidats qu'ils lui présenteraient, gratuitement et sans exiger un serment d'allégeance, cet accord qui satisfait probablement tous les maîtres est une victoire incontestable pour la frange la plus modeste d'entre eux, désormais affranchie de cette dépense supplémentaire et de la marque de sujétion à l'égard du chancelier.

Fragmentairement mises en lumière par l'étude prosopographique, les origines géographiques et sociales des maîtres de la "première génération» universitaire ne suffisent naturellement pas à expliquer à elles seules la genèse de l'Université, que l'historiographie a pu à juste titre associer à des facteurs politiques, intellectuels, sociaux, ecclésiastiques voire religieux. Toutefois, comme les sources le révèlent parfois de façon allusive, ce sont bien ces maîtres, majoritairement anglais ou nés en France septentrionale, anciens étudiants de Bologne, formés au trivium et à la théologie, clercs nobles ou bourgeois, fils d'artisans parfois modestes, qui ont en assemblée rédigé de premiers statuts et réglementé au mieux l'institution naissante, qui ont lutté contre l'hégémonie du chancelier; ils ont été les principaux acteurs du processus. On a vu que la diversité de leurs expériences en France comme hors du royaume de France, la palette de leurs apprentissages antérieurs comme la variété de leurs modes de vie ou de leurs valeurs ont assurément modelé l'institution

\footnotetext{
${ }^{61}$ Chronica majora Rogeri de Hovedene, éd. W. Stubbs, vol. IV, Londres, 1871, p. 120.

${ }^{62}$ J. Verger, "Les conflits town and gown au Moyen Âge. Essai de typologie », dans Les universités et la ville au Moyen Âge, éd. P. Gilli, D. Le Blévec, J. Verger, Leyden, 2007, p. 237-255.

${ }^{63}$ J. Verger, dans son article «À propos de la naissance de l'Université » (cit. note 2), rappelle qu'au début, l'Université est avant tout une association d'entraide. Ces exigences viennent aussi, semble-t-il, de pratiques canoniales et monastiques.
} 
qu'ils ont inventée, ont orienté des choix institutionnels propres à Paris, mais d'une manière qui reste, faute de documentation, bien difficile à déterminer plus précisément.

Nathalie Gorochov Université Paris 12 Val-de-Marne

CREPHE (EA 2352) 


\section{Annexe : Brèves notices des maîtres présents à l'université de Paris entre 1200 et 1231}

Ces informations sont indicatives et, en aucun cas, exhaustives.

Abréviations :

- Baldwin : J. W. Baldwin, «Masters at Paris 1179-1215», dans Renaissance and Renewal in the Twelfth Century, éd. R. L. Benson et G. Constable, Cambridge, 1982, p. 138-172.

- Cardinali : A. Paravicini-Bagliani, dans Cardinali di Curia e familiae cardinalizie dal 1227 al 1254, 2 vol., Padoue, 1972.

- Chartres : E. de Lépinois et L. Merlet, Cartulaire de Notre-Dame de Chartres, 3 vol., Chartres, 1863-1865.

- Chartularium : H. Denifle et E. Châtelain, Chartularium Universitatis Parisiensis, t. 1 (1200-1289), Paris, 1889. 1895.

- Clerval : E. Clerval, Les écoles de Chartres du $V^{e}$ au XVI siècle, Chartres,

- DLF : Dictionnaire des lettres françaises. Le Moyen Age, nouv. éd. G. Hasenohr et M. Zink, Paris, 1992.

- Emden : A. B. Emden, A Biographical Register of the University of Oxford to A.D. 1500,3 vol., Oxford, 1957.

- Fasti Reims : P. Desportes, Fasti Ecclesiae Gallicanae, t. 3 : Église de Reims, Turnhout, 1998.

- MT1 ou MT2: P. Glorieux, Répertoire des maîtres en théologie de Paris au XIII siècle, Paris, 2 vol., 1933.

- Helvetia : Helvetia Sacra. Section I: Archidiocèses et diocèses, t. 3 (Genève) et t. 4 (Lausanne).

- Lefebvre 2001: A. Lefebvre-Teillard, «Petrus Brito legit... Sur quelques aspects de l'enseignement du droit canonique à Paris au début du XIII ${ }^{\mathrm{e}}$ siècle », Revue d'histoire du droit, 79, 2, avril-juin 2001, p. 153-177.

- Lefebvre 2002: A. Lefebvre-Teillard, "Magister A. Sur l'école de droit canonique parisienne au début du $\mathrm{XIII}^{\mathrm{e}}$ siècle », Revue d'histoire du droit, 80, 4, octobre-décembre 2002, p. 401-417.

- Maleczek: W. Maleczek, Papst und Kardinalskolleg von 1191 bis 1216, Vienne, 1984.

- Pycke : J. Pycke, Répertoire biographique des chanoines de Tournai 10801300, Louvain, 1988.

- Russell : J. C. Russell, «Dictionary of writers of XIIIth Century England », Bulletin of the Institute of Historical Research. Supplement, 3, 1936.

-Wickersheimer: E. Wickersheimer, Dictionnaire biographique des médecins en France au Moyen Age, Paris, 1936, réédition et supplément par D. Jacquart, Genève, 1979.

MAITRE A : maître en décret entre 1205 et 1211 (Lefebvre 2002). 
ADAM OSB : anglais, a étudié à Oxford, vient à Paris en 1209 (Emden, p. 12).

ADAm DE Courlandon : a enseigné la théologie à Paris dans le premier quart du XIIIe siècle, est apparenté aux seigneurs de Courlandon, serait le frère de Renaud de Courlandon $(† 1207)$ (W. M. Newman, «Les seigneurs de Nesle en Picardie $\left(\mathrm{XII}^{\mathrm{e}}-\mathrm{XIII}{ }^{\mathrm{e}}\right.$ siècles) ", Memoirs of the American Philosophical Society, 91, 1971, p. 140-144).

AdAm De Pulchre Mulieris : maître en théologie vers 1210 (MT1, p. 288).

AlAin DE BEECLES : anglais, maître dès 1201, enseigne probablement à Paris jusqu'en 1229, puis repart pour l'Angleterre (Emden, p. 145).

AleXANDRE De Hales : anglais, maître ès arts à Paris à partir de 1209-1210 environ, riche selon Bonaventure (Emden, p. 2180).

AleXANDRE DE VILLEDIEU : normand, étudie puis enseigne les arts à Paris fin du XII ${ }^{\mathrm{e}}$ début du XIII ${ }^{\mathrm{e}}$ siècle (DLF, p. 48-49).

AMAURY DE BENE : maître ès arts parisien avant 1210 (E. Gilson, La philosophie au Moyen Âge, Paris, 1962, p. 382-384).

ANDREAS SUNESON : maître en théologie à Paris vers 1200, son père Sune Ebbesen est l'un des plus riches seigneurs Danois (Baldwin).

ARDENGUS : italien, maître résidant à Paris entre 1227 et 1230 (MT1, p. 284).

ARNOUL DE LA PIERRE : demi-frère de Richard de Fournival, enseigne la théologie à Paris vers 1220-1230 (MT1, p. 302).

AUBRY CORNUT : noble, étudie les arts, les deux droits puis la théologie à Paris début XIII ${ }^{\mathrm{e}}$ siècle (Chartres, t. 3, p. 197).

BARTHELEMY : de Chartres, enseigne le droit à Paris (Lefebvre 2002).

BARTHELEMY L'ANGLAIS OFM: enseigne la théologie à Paris au couvent des Mineurs en 1231 (Russell, p. 21-22, B. Ribémont, préface au Livre des propriétés des choses, Paris, 1999).

BARTHELEMY DE MONCY : maître en droit parisien vers 1220-1230 (Chartres, t. 3, p. 222).

Benedictus de SAwston : anglais, enseigne le droit à Paris en 1214 (S. Kuttner et E. Rathbone, «Anglo-Norman Canonists of the XIIth century », Traditio, 1949-1951, p. 279358). 79).

BernARD CHABERT : maître en théologie, chancelier (1205) (Helvetia, vol. 3, p. 78-

Boniface Clutinc de BruXelles : de la famille patricienne des Clutinc, étudie et enseigne à Paris entre 1200 et 1215 (Helvetia, vol. 4, p. 119 ; C. Renardy, Les maîtres universitaires dans le diocèse de Liège. Répertoire biographique, Paris, 1981, p. 199-200).

CONON D'ESTAVAYER: fils des sires d'Estavayer le Lac, fait plusieurs séjours à l'université de Paris, entre 1212 et 1215 d'abord, en 1222-1223 également (Helvetia, vol. 4, p. 375-376).

DAVID DE DinANT : maître ès arts au début du XIII ${ }^{\mathrm{e}}$ siècle (Gilson, op. cit., p. 384-5).

DReuX DE HAUTVILliers : maître ès arts, docteur en lois (Fasti Reims, p. 263).

DUDO : maître, prêtre, amauricien, condamné en 1210 (Chartularium, p. 70).

EDMOND D'ABINGDON : anglais, fils de Reginald d'Abingdon le Rich, peut-être un marchand, qui possède plusieurs maisons (C. H. Lawrence, Saint Edmund of Abingdon. A Study in Hagiography and History, Oxford, 1960, p. 106 ; Emden, p. 6-7).

ÉTIENNE BEROUT : parisien, il est déjà maître ès arts de l'université de Paris en 1209 quand son père Étienne Bérout, bourgeois de Paris, fonde un collège (Chartularium, p. 68-69). Plus tard, maître en théologie (MT1, p. 295). 
ÉTIENNE CUDOT : maître ès arts vers 1220, maître en théologie dès 1230 (MT1, p. $321)$.

ÉTIENNE DE GRES : du Chartrain, juriste (Chartres, t. 3, p. 51).

ÉTIENNE LANGTON : noble anglais, son père Henri Langton est «a country gentleman of modest standing in Lincolnshire », a un manoir. Maitre en théologie à Paris jusqu'en 1206 (F. M. Powicke, Stephen Langton, Oxford, 1928, p. 4 sq.).

ÉTIENNE DE LEXINGTON : noble, plus jeune fils de Richard de Lexington, qui tient son nom des manoir et paroisse de Lexington dans le comté de Nottingham; étudie les arts à Paris entre 1200 et 1215 environ où il est l'élève d'Edmond d'Abingdon (C. H. Lawrence, «Stephen de Lexington and Cistercian University Studies in the XIIIth Century », Journal of Ecclesiastic History, 11, 1960, p. 164-178).

ÉTIENNE DE REIMS : maître ès arts puis en théologie dès 1210 (MT1, p. 271).

ÉTIENNE DE Provins : maître ès arts puis en théologie vers 1230 (MT1, p. 303).

Eudes De Chateauroux : né à Châteauroux, étudiant en arts à Paris vers 1210, puis en théologie vers 1215, accède au titre de maître en théologie dès 1229 (A. Charansonnet, «Du Berry en Curie : la carrière du cardinal Eudes de Châteauroux (1190 ?-1273) et son reflet dans sa prédication », Revue d'histoire de l'Église de France, 2000, p. 5-37).

EUDES DE CHERITON : noble anglais, les Cheriton portent le nom de leur manoir dans le Kent, étudiant en arts puis en théologie à Paris entre 1200 et 1220 au moins (Albert C. Friend, «Master Odo of Cheriton », Speculum, 1948, p. 641-658).

FEDERICO VISCONTI : né vers1200, membre de la famille aristocratique pisane des Visconti Ricoveranza, probablement maître ès arts, il étudie la théologie à Paris entre 1220 et 1230, puis après 1236 (N. Bériou et I. Le Masne de Chermont, Les sermons et la visite pastorale de Federico Visconti, archevêque de Pise, Rome, 2001, introduction).

G. DE LA MARE : maître parisien attesté en août 1213 (Chartularium, $\mathrm{n}^{\mathrm{O}}$ 16).

GAUtier L'ANGLAis : maître ès arts et poète enseignant sur le Petit-Pont dans la première moitié du XIII ${ }^{\mathrm{e}}$ siècle (Russell, p. 174).

GAUTIER CORNUT : noble, frère d'Aubry Cornut, originaire du Chartrain, a enseigné à Paris dans le premier quart du XIII ${ }^{\mathrm{e}}$ siècle, d'après Jean de Garlande qui le cite parmi les professeurs fameux de Paris (Clerval, p. 325).

GAUTIER DE MARVIS : fils de Gautier d'Allain, bourgeois de Tournai, maître ès arts avant 1205, encore à Paris en 1210 (Pycke, p. 138-139).

Gautier (Walter) DE Suffeld : maitre en décret de Paris vers 1230 (Emden, p. 1813-1814).

Gautier Teutonique OP : maître ès arts, parti à Verceil, entre dans l'ordre des Prêcheurs en 1229 (Chartularium, p. 131-132).

GAUTIER DE WANGIERMES : fils du bourgeois de Tournai Jacques de Wangiermès, étudiant puis maître ès arts à Paris entre 1220 et 1230 (Pycke, p. 89-91).

GeOFFroy DE Poitiers : élève d'Étienne Langton à Paris avant 1206, encore attesté comme maître en théologie en 1231 (MT1, p. 298).

GERVAIS DE MELCHELEY : anglais, poète et grammairien, élève puis collègue de John Blund à Paris entre 1200 et 1220 environ (Emden, p. 1256).

GILLES DE CORBEIL : d'origine modeste, maitre en médecine sous le règne de Philippe Auguste (C. Vieillard, Essai sur la société médicale et religieuse au XIIe siècle. Gilles de Corbeil, Paris, 1909 ; Wickersheimer, p. 196-197).

MAITRE GIRARD : maître en décret entre 1205 et 1211 (Lefebvre 2001). 
GREGoire DEI CRescenZi : famille noble romaine des Crescenzi, neveu de cardinal, maître parisien entre 1200 et 1216 , date à laquelle il est promu cardinal par Innocent III (Malezcek, p. 183-184)

GuerRic De SAINT-Quentin OP : originaire du Vermandois, maitre ès arts de Paris, entre chez les Dominicains vers 1225 et entreprend des études en théologie, part à Bologne vers 1230 puis revient à Paris (MT2, p.54).

GUI D'ORCHELLES : picard, il est en 1210 un jeune maître ès arts qui commence des études en théologie, devient maître en théologie entre 1220 et 1223 (MT1, p. 285).

GUIARD DE LAON : issu de la petite noblesse du Laonnois, étudiant en théologie à Paris, licencié vers 1210 (P. Boeren, Les æuvres et la vie de Guiard de Laon 1170 env.-1248, La Haye, 1956, p. 16).

Guillaume D'Auvergne : maître ès arts vers 1210, maître en théologie dès 1223 (MT1, p. 315).

Guillaume D'AuXerre : maître en théologie à Paris en 1228 (MT1, p. 293).

Guillaume De Durham : anglais, maître en théologie de Paris entre 1226 et 1229 (Emden, p. 612-613).

Guillaume De Joinville: noble, maître en théologie de Paris jusqu'en 1208, plus tard archevêque de Reims (Fasti Reims, p. 158-159).

Guillaume De PARIS OU De Chartres : originaire de Paris, il est maître ès arts, puis étudie le droit canon et la théologie dans les années 1220 (Clerval, p. 329).

Guillaume De Poitiers : partisan d'Amaury de Bène, maitre ès arts et étudiant en théologie en 1210 (Chartularium, p. 70).

Guillaume de Pont DE L'ArChe : normand, maître en théologie en 1218 (MT1, p. 279).

Guillaume De SAINT-AmOuR : a des parents pauvres selon son testament ; quitte son village natal du Jura pour étudier à Paris vers 1215 probablement, et il est maitre ès arts au plus tard en 1230 (M.-M. Dufeil, op. cit., p. XXI-XXII).

Haymo de FAVERsham OFM : anglais, maittre de Paris, entre chez les Franciscains en 1224 (Emden, p. 671-672).

HenRI D'AvRANCHES : maître ès art de Paris vers 1214 (Emden, p. 80-81).

HENRI DE BURFORD OFM: maître anglais présent à Paris dans les années 1220 (Russell, p. 44).

HENRI DE GRES : maître en droit avant 1230 (Clerval, p. 325).

HenRi DE RERESBy OFM : anglais, maître à Paris entre 1210 et 1229 (Emden, p. 1567).

Hugues DE SAINT-CHER OP : peut-être né dans une famille noble vassale des ducs de Savoie, vient étudier à Paris vers 1210 ; maître ès arts, il commence des études en théologie ; vers 1225, alors qu'il est bachelier, il prend l'habit dominicain et enseigne la théologie au moins jusqu'en 1233 (MT2, p. 43).

Humbert De Pirovano: de la famille milanaise des Pirovano, qui a compté plusieurs archevêques de Milan avant 1200, maître en théologie à Paris de 1200 à 1206, devient à cette date archevêque de Milan puis cardinal (Baldwin; Malezcek p. 183-184).

JACQUES D'ARRAS : maître en théologie, selon toute vraisemblance a étudié puis enseigné entre 1200 et 1220 à Paris (MT1, p. 286).

JACQUES DE DINANT : maître ès arts dès 1228 (Chartularium, p. 117), devient maître en théologie avant 1243 (MT1, p. 326). 
Jacques Pantaleon D'Ancher (ou De Troyes) : fils d'un cordonnier de Troyes, va étudier à Paris les arts libéraux et le décret - entre 1200 et 1215 environ - où il obtient probablement le grade de maître (W. Sievert, «Das Vorleben des Papstes Urban IV. Beilage. Die Biographien des Papstes Urban IV von Gregor Segni und Thierricus Vallicolor », Römische Quartalschrift, 10, 1896, p. 451-505 et 12, 1898, p. 127-161 ; Agostino Paravicini Bagliani, "Gregorio di Napoli, biografo di Urbano IV », Römische historische Mitteilungen, 11, 1969, p. 59-78; Jacques Foviaux, «Les sermons donnés à Laon en 1242 par le chanoine Jacques de Troyes, futur Urbain IV », Recherches augustiniennes, 20, 1985, p. 203-256).

JOHN BLuND: anglais, il a étudié les arts à Paris et il est peut-être reparti à Oxford après sa maîtrise ès arts. Plus tard, il étudie la théologie à Paris, entre 1208 et 1220 , enseigne la théologie à Paris jusqu'en 1229 (Emden, p. 206).

Jean de Barastre (ou De SAint-Quentin ou De SAint-Albans) : anglais, maître en théologie entre 1200 et 1220 au moins (MT1, p. 274).

JEAN DE CANDELIS : devient chancelier de Paris début 1210, alors qu'il est déjà maître en théologie, reste chancelier jusqu'en 1214 (MT1, p. 270).

JEAN DE GARLANDE : maître ès arts de Paris dans le premier tiers du XIII ${ }^{\mathrm{e}}$ siècle (DLF, p. 779-780).

JEAN (HALGRIN) D'ABBEVILLE : peut-être membre de la famille nobiliaire picarde des Halgrin, étudiant puis maître en théologie entre 1200 et 1216 au moins (MT1, p. 272-273 ; Cardinali, p. 21).

JEAN DE LA ROCHELLE OM : maître ès arts, étudiant en théologie, franciscain vers 1228 (MT2, p. 25).

JEAN DE MATHA : enseigne la théologie à Paris au début du XIII ${ }^{\mathrm{e}}$ siècle (Baldwin).

JEAN PAGUS : maître ès arts en 1231 (MT1, p. 328).

JEAn DE SAINT-Gilles OP : anglais, a étudié la médecine à Paris et Montpellier, est devenu maître en médecine puis a étudié la théologie à Paris. Entré dans l'ordre dominicain en 1230 (Russell, p. 73-75; MT2, p. 52-53).

LAURENT DE Fougeres : avec Gilles de Corbeil, seul maître parisien attesté de manière certaine avant 1231 (Wickersheimer, p. 520).

MARTIN : maître en théologie vers 1200 (Baldwin).

MATHIEU D'ÉCOSSE : maître en théologie en 1219 (Chartularium, p. 85).

NicolAS D'AMIENS DE BADIANDO : maître en théologie vers 1200 (MT1, p. 263)

Nicolas (BuchiAu) DE TOURnAi: membre de la famille patricienne de Buchiau, maître ès arts puis en théologie entre 1200 et 1218 (Pycke, p. 22 à 25).

NiCOLAS DE CANNIS : noble, maître, « instruit dans les deux droits », a étudié le droit à Paris vers 1220-1230 (Chartres, t. 3, p. 61).

NiCOLAS DE FARNHAM : anglais, probablement noble. Il a pendant plusieurs années enseigné à la faculté des arts de Paris, enseigné la médecine à Bologne, puis a étudié la théologie à Paris (Emden, p. 669).

Nicolas DE FlaVignY: en 1210, étudiant à Paris, maître ès arts puis maître en théologie avant 1227 (MT1, p. 287).

Nicolaus HAUdRI DE ChARTREs : fils de bourgeois de Chartres, à Paris vers 1210 pour étudier et enseigner les arts, puis étudie la médecine à Montpellier, enseigne le droit canon à Bologne, puis revient à Paris pour y étudier la théologie (Chartres, t. 3, p. 179).

MaItRe P : enseigne le décret entre 1205 et 1210 (Lefebvre 2001).

MAITRE Petrus BRITo : enseigne le décret entre 1205 et 1218 (Lefebvre 2001). 
Philippe le Chancelier : fils illégitime de l'archidiacre de Paris Philippe, il obtient la licence en théologie vers 1206-1207, chancelier de l'Église de Paris entre 1218 et 1236 (MT1, p. 282).

Philippe De Greve: fils de Philippe de Grève bourgeois de Paris (Molinier, Obituaires, t. 1, p. 129), maître ès arts dans le premier quart du XIII ${ }^{\mathrm{e}}$ siècle.

PIERRE DE BAR-SUR-AuBE : membre de la famille des comtes de Bar-sur-Aube, maître parisien vers 1230 (Cardinali, p. 213-217).

PIERRE DE CAPOUE : noble italien, maître en théologie entre 1201 et 1219 , promu cardinal (MT1, p. 265-266).

Pierre Petit : maître en théologie dès 1209, chancelier de Paris en 1244 (MT1, p. 312)

Pierre PeVerel: peut-être anglais, maitre en décret entre 1209 et 1214 (Chr. Coppens, «The Teaching of Law in the University of Paris in the First Quarter of the XIIIth Century ", Rivista internazionale del diritto comune, 10, 1999, p. 139-173).

PierRe DE Poitiers : maître en théologie et chancelier de 1193 à $1205(\mathrm{Ph}$. S. Moore, The Works of Peter of Poitiers, Notre Dame, Indiana, 1936).

PIERRE DE ROISSY : maître en théologie entre 1200 et 1205 (Clerval, p. 327).

Prevotin: de Crémone, est maître en théologie et chancelier de l'Église de Paris entre 1206 et 1210 (G. Lacombe, Prepositini cancellarii Parisiensis (1206-1210), Opera Omnia, t. 1 : La vie et les œeuvres, Paris, 1927, p. 46).

RAOUL ARDENT : né près de Bressuire, enseigne la théologie (Baldwin).

RAOUl DE CORBRIGGE OFM : anglais, étudie la théologie à Paris vers 1230, y devient maître en théologie (Emden, p. 484).

Raoul De Maidstone : maître en théologie parisien, quitte Paris en 1229 (Emden, 1203-1204).

Renaud D'orleans OP : compagnon de saint Dominique, maitre en décret de Paris entre 1205 et 1211 (Lefebvre 2002)

RICHARD ARCHIDIACRE D'ÉVREUX: maître ès arts, étudiant la théologie en 1222 (Chartularium, p. 104).

RichaRd DE Fournival : fils de Roger de Fournival, médecin de Philippe Auguste, maître à Paris début XIII ${ }^{\mathrm{e}}$ siècle (Cardinali, p. 138-140).

RICHARD LE GRANT WEATHERSHED : anglais, maître en théologie entre 1200 et 1218 (Emden, p. 2188).

RiCHARD DE MORES (Baldwin) : maitre en décret, ses études et son enseignement à Paris et Bologne datent d'avant 1202. Revient à Paris en 1215 pour étudier la théologie (Russell, p. 111-113).

RICHARD POORE : anglais, élève d'Étienne Langton fin XII ${ }^{\mathrm{e}}$ siècle à Paris, de nouveau à Paris de 1208 à 1214 (Russell, p. 118-119).

RichARD RUfuS DE CORNOUAILlES OFM : ce maitre ès arts anglais de Paris, étudiant la théologie, prend l'habit franciscain en 1229, fait profession à Oxford puis revient à Paris pour étudier la théologie (Emden, p. 1604-1605).

ROBERT D'ABINGDON : anglais, frère d'Edmond, l'accompagne à Paris dans le premier quart du XIII ${ }^{\mathrm{e}}$ siècle (Emden, p. 8).

ROBERT BACON : anglais, il étudie la théologie auprès de maitre Jean d'Abbeville à partir de 1208-1209 (Emden, p. 87).

RoBERT BLUND : oncle de John, enseigne à Paris jusque vers 1200 (Emden, p. 126). 
Robert Du CoIn-Du-Mur : maître en décret de Paris avant 1230 (Chartres, t. 3 p. 131-132).

ROBERT DE COURSON : appartient à une noble famille anglaise du Derbyshire, maître en théologie entre 1200 et 1212, devient cardinal en 1212 (Emden, p. 498-499; M. et C. Dickson, «Le cardinal Robert de Courson, sa vie », Archives d'histoire doctrinale et littéraire du Moyen Âge, 9, 1934, p. 53-142 ; Baldwin, Masters, Princes and Merchants, p. 19 sq.).

ROBERT DE DOMFronT : maître, écolâtre de Paris, chanoine du Mans dès les années 1220 résidant à Paris (Registres de Grégoire IX, $\mathrm{n}^{\mathrm{0}} 1088,1136$ ).

ROBERT DE SOMERCOTES : apparenté à la famille des Foliot, frère du juriste Laurent de Somercotes (Cardinali, p. 132); il a étudié à Bologne et à Paris avant 1232 (Emden, p. 22162217).

Robert DE SORBon : fils de «vilains » (Joinville), vient étudier les arts à Paris, puis maître ès arts sans doute vers 1220, étudie la théologie jusque vers 1240, devient maître en théologie (MT1, p. 340).

Rodoicus Modicipassus: maître en décret à Paris attesté entre 1204 et 1207 (Baldwin).

Roger (PARvus) DE SAlisbury: anglais, qui a des biens à Oxford; étudiant en théologie à Paris entre 1200 et 1223 (Emden, p. 1632).

RolAND DE CREMONE OP : maître ès arts de Bologne, y prend l'habit ; vient étudier la théologie à Paris, premier maître en théologie dominicain de Paris en 1229 (MT2, p. 42).

SIMON D'AUTHIE : maître ès arts et bachelier en théologie avant 1229, attesté comme maître en théologie en 1231 (MT1, p. 312).

SiMON LANGTON : anglais, noble, frère d'Étienne, maître ès arts, théologien, chanoine de Paris à partir de 1206. Se trouve à Paris pendant l'Interdit comme son frère Étienne (Russell, p.152-154).

THIBALDUS : maître en décret de Paris 1205-1211 (Lefebvre 2002).

ThOMAS DE CHOBHAM : anglais, vient étudier les arts puis la théologie à Paris vers 1190, enseigne à Paris de nouveau entre 1222 et 1228 comme maître en théologie (F. Morenzoni, Des écoles aux paroisses. Thomas Chobham et la promotion de la prédication au début du XIIIe siècle, Paris, 1995, p. 14-21).

Thomas Gallus : chanoine de Saint-Victor, maître en théologie dans le premier quart du XIIIe siècle (MT1, p. 277-8).

ThOMAS DE SALISBURY : maître présent à Paris avant 1220 (Russell, p. 169).

W. De SANCto Melano : maître parisien en 1213 (Chartularium, $\mathrm{n}^{\circ}$ 16). 